Didáctica. Lengua y literatura

ISSN: 1130-0531

http://dx.doi.org/10.5209/DIDA.61951

\title{
Los modelos de género discursivo en la planificación y revisión de una reseña de lectura
}

\author{
Victoria Abad Beltrán ${ }^{1}$; Carmen Rodríguez Gonzalo²
}

Recibido: 3 de noviembre de 2016 / Aceptado: 18 de julio de 2017

Resumen. En este trabajo se analiza la incidencia de los modelos de género discursivo en la planificación y revisión de la escritura desde una intervención educativa cuyo diseño sigue el modelo de secuencias didácticas para aprender a escribir. La secuencia diseñada tenía como objetivo que alumnos de $3^{\circ}$ ESO elaboraran una reseña de lectura y para ello ofrecía diferentes modelos de este género. El análisis de los datos del proceso (textos intermedios e interacciones) y del producto (textos elaborados por los alumnos) permite vincular el uso de modelos de género, las operaciones de planificación y revisión y la apropiación de los estereotipos (representaciones mentales arraigadas en una dimensión social e histórica).

Palabras clave: Géneros discursivos; enseñanza de la escritura; revisión textual; planificación textual; secuencias didácticas.

\section{[en] Discursive Genre Models in the Planning and Revision of a Reading Review}

\begin{abstract}
This work questions the incidence of genre discourse models in the planning and revision of writing from an educational intervention whose design follows the model of teaching sequences to learn to write. The objective of the designed sequences was that the students write a reading review and so it offers different models of this genre. The analysis of process data (intermediate texts and interactions) and product data (texts produced by the students) link the use of genre models, planning and review operations and appropriation of stereotypes (mental representations rooted in a social and historical dimension).
\end{abstract}

Keywords: Speech genres; teaching writing; text revision; textual planning; teaching sequences.

\section{[fr] Modèles de genre discursif dans la planification et la révision d'une recension}

Résumé. Dans cet article, l'influence des modèles de genre discursive dans la planification et la révision est analysée à partir d'une intervention éducative dont la conception suit le modèle des séquences d'enseignement pour apprendre à écrire. Le but de la séquence conçue était que les étudiants de $3^{\text {e }}$ ESO écrivent une recension. Ainsi, elle offre modèles de ce genre. L'analyse des données du processus (intermédiaires textes et interactions) et du produit (textes produits par les étudiants) lie l'utilisation de

\footnotetext{
1 GIEL-IES Camp de Morvedre. Puerto Sagunto, Valencia (España)

E-mail: victoriaabadbeltran@yahoo.es

2 GIEL-Departament de Didàctica de la Llengua i la Literatura. Universitat de València (España)

E-mail: crodrig@uv.es
} 
modèles, la planification et la révision et l'appropriation des stéréotypes (des représentations mentales enracinées dans une dimension sociale et historique).

Mots-clé: Genres discursives ; enseignement de l'écriture ; révision des textes ; planification textuelle ; séquences d'enseignement.

Sumario. 1. Introducción. 2. Marco teórico. 3. Objetivos. 4. El derecho como cultura. 5. Metodología. 5.1. Las operaciones de escritura y el uso de las ayudas de la SD. 5.2. Las transformaciones textuales y el uso de ayudas durante el proceso. 5.3. El proceso de apropiación del género. 6. Conclusiones. 7. Bibliografía.

Cómo citar: Abad Beltrán, V.; Rodríguez Gonzalo, C. (2018). Los modelos de género discursivo en la planificación y revisión de una reseña de lectura, Didáctica. Lengua y literatura, 30, 11-21.

\section{Introducción}

En la actualidad está consolidada la idea de que la enseñanza de la escritura ha de tener un enfoque social, por lo que el objetivo ha de ser que los alumnos aprendan a participar en interacciones de diferentes contextos. El aprendizaje de la escritura se define, pues, como la apropiación de una gran diversidad de géneros discursivos (Abril Villalba, 2006; Álvarez Angulo, 2006; Álvarez Angulo y García Parejo, 2010; Amo y Ruiz Domínguez, 2006; Camps y Ribas, 2000; Zayas, 1997 y 2012, entre otros).

Para conseguir que los alumnos se apropien de estos discursos, los proyectos de escritura incluyen textos modelo del género seleccionado. No obstante, en la práctica de aula se observa que el uso que hacen los alumnos de los modelos de género no coincide en muchas ocasiones con los objetivos del docente y que algunos alumnos tienen dificultades para establecer vínculos entre los modelos y los textos que crean.

En este artículo presentamos una investigación (Abad, 2015) que indaga en la incidencia de los modelos de género discursivo en el proceso de escritura. Para ello, partimos de un proyecto de escritura cuyo objetivo es que los alumnos elaboren una reseña de lectura y nos centramos en analizar cómo los estudiantes usan los modelos de este género para comprender las operaciones mentales realizadas durante la tarea y las interacciones que se producen en el aula entre los alumnos y estos materiales.

\section{Marco teórico}

Los modelos sociocognitivistas consideran las interacciones entre los individuos y entre los individuos y su contexto social como elementos potenciadores del aprendizaje. Estos estudios conciben la escritura como "un fenómeno complejo en el que intervienen aspectos antropológicos, sociales, cognitivos, lingüístico-textuales y didácticos, en cuanto que nos preocupa el sujeto que aprende y el individuo que enseña [...] en una determinada sociedad" (Álvarez Angulo, 2010, 20).

Como es sabido, el proceso de escritura se inicia en el momento en el que el escritor se hace una representación de la tarea y está compuesto por las operacio- 
nes de planificación, de textualización y de revisión (Flower y Hayes, 1980). Estas operaciones son recursivas porque en la planificación se establecen unos criterios para revisar y se elabora una representación mental del texto (Scardamalia, Bereiter y Goelman, 1982; Castelló, 2000) y porque durante la revisión puede reformularse la planificación inicial. La revisión, por tanto, no se daría solo en la fase final de la escritura sino a lo largo de todo el proceso.

Además, como la revisión textual es una actividad compleja y difícil, porque supone tratar en tiempo real problemas de diferentes niveles y porque requiere un distanciamiento del texto, se deben tomar decisiones para reducir esta complejidad y focalizar la atención en un aspecto del texto o en un modo de evaluarlo. En este sentido, Ganier (2006) distingue dos procesos para jerarquizar las estrategias de revisión: un orden determinado por la linealidad textual (es la jerarquización más común entre los escritores no experimentados) y un orden focalizado en niveles lingüísticos (el escritor selecciona las estrategias de revisión teniendo en cuenta la globalidad del texto y sus aspectos estructurales).

La teoría de los prototipos nos puede ayudar a entender los procesos cognitivos que se llevan a cabo en la comprensión y expresión escrita. Esta teoría, siguiendo a Cuenca y Hilferty (1999), establece que las categorizaciones son mecanismos que posibilitan organizar la información que recibimos y que se establecen por los procedimientos de generalización (para estructurar la información que recibimos) y de discriminación (para diferenciar los elementos). Dentro de cada categoría hay unos elementos más prototípicos, es decir, más representativos (la imagen mental de esa categoría), y otros elementos más periféricos. Entre unos y otros hay una gradación. En ocasiones sucede que la categorización de la realidad no se establece por elementos prototípicos sino por efectos de prototipicidad, por interrelaciones imperfectas entre la realidad y un modelo cognitivo idealizado.

Los estereotipos también serían representaciones o imágenes mentales preconstruidas, relativamente fijadas y frecuentes (Kervyn, 2009), pero se diferencian de los prototipos en que se enraizan en una dimensión histórica y social. Los prototipos explican cómo las personas categorizan la realidad que perciben, mientras que los estereotipos vinculan estas categorizaciones con la memoria colectiva de una comunidad y con la sociedad. De ahí la potencialidad didáctica del estereotipo: es una vía de acceso al saber, cercana a los alumnos, a partir de la cual se pueden articular secuencias didácticas que permiten una apropiación de saberes más complejos.

\section{Objetivos}

En relación con la enseñanza de la escritura esta investigación se plantea el objetivo general de entender la incidencia de los modelos de género (en este caso, de modelos de reseñas de lectura) en los procesos de escritura. En relación con este objetivo general, se desglosan dos objetivos más específicos. En primer lugar, estudiar cómo el uso de estos modelos afecta a las operaciones de escritura y, en segundo lugar, establecer en qué sentido los modelos pueden contribuir a la construcción del estereotipo. 


\section{Metodología}

La investigación que presentamos es cualitativa, con tratamiento cuantitativo de los datos extraídos a partir de la aplicación de una secuencia didáctica (SD) en una situación natural de aula (Rodríguez Gonzalo, 2008 y 2009). La SD tenía como objetivo la escritura de una reseña de lectura con la ayuda de diversos modelos de reseña, de una plantilla de planificación que permitía a los alumnos dividir su texto en tres partes (en la primera parte se seleccionaba información relevante de la novela, en la segunda se valoraba la obra de lectura y en la última se recomendaba su lectura -véase la figura 7-) y de una ficha de revisión que elaboró el grupo-clase guiado por la profesora.

La propuesta de escritura se llevó a la práctica en dos aulas de $3^{\circ} \mathrm{ESO}^{3}$ durante ocho sesiones, divididas en cuatro fases (véase figura 1). El análisis se centra en la última fase, en la que los alumnos se enfrentaban al proceso de elaboración textual, esto es, a las operaciones de planificación, textualización y revisión. En las fases previas a la elaboración de la reseña, los alumnos habían realizado diferentes actividades de análisis de modelos del género centradas en el contexto comunicativo de estos textos y en sus características estructurales y lingüísticas. La escritura de la reseña se llevó a cabo por grupos homogéneos de dos o tres alumnos formados por la profesora. Para el análisis de datos se seleccionaron cinco grupos. Estos datos provenían de diferentes fuentes:

1. Grabaciones en audio de las interacciones orales producidas mientras los alumnos elaboraban su reseña de lectura. En estas grabaciones aparecen, por un lado, las interacciones de la profesora con el grupo-clase y con el grupo de escritura y, por otro, las interacciones entre iguales. Estas interacciones ofrecen datos para desvelar qué procesos cognitivos siguen los alumnos y cómo usan los modelos de reseña que aparecen en la SD.

2. Textos intermedios y textos finales creados por los alumnos: estos textos permiten mostrar el proceso de apropiación del estereotipo y analizar las transformaciones textuales (Allal, 2000), término en el que incluimos las reformulaciones (Camps, Guasch, Milian y Ribas, 2007) y las revisiones que introducen los alumnos en sus escritos. Estas transformaciones aparecen clasificadas en transformaciones profundas (las que afectan a la superestructura, al texto en su globalidad y a los aspectos de la escritura en los que se tiene en cuenta el receptor y la intencionalidad), y transformaciones superficiales (las que afectan al contenido semántico, a la ortografía y a la cohesión del texto o la frase).

En el sistema educativo español la educación secundaria obligatoria (ESO) está dividida en cuatro cursos. Se inicia en $1^{\circ}$ ESO (con 12 años) y finaliza en $4^{\circ}$ ESO (con 16 años). Los alumnos que formaron parte de este proyecto de escritura eran de $3^{\circ}$ ESO (14 y 15 años). 


\begin{tabular}{|c|c|c|c|}
\hline $\begin{array}{l}\text { FASE } 1 \\
\text { Escritura de un } \\
\text { texto inicial }\end{array}$ & $\begin{array}{l}\text { FASE } 2 \\
\text { Presentación y repre- } \\
\text { sentación de la tarea }\end{array}$ & $\begin{array}{l}\text { FASE } 3 \\
\text { Análisis de modelos }\end{array}$ & $\begin{array}{l}\text { FASE } 4 \\
\text { Proceso de elabora- } \\
\text { ción textual }\end{array}$ \\
\hline $\begin{array}{l}\text { Actividad previa } \\
\text { de escritura de una } \\
\text { reseña sin regula- } \\
\text { ciones externas }\end{array}$ & $\begin{array}{l}\text { Actividades de interac- } \\
\text { ción oral guiadas por la } \\
\text { profesora }\end{array}$ & $\begin{array}{l}\text { - Actividades } 1 \text { y } 2 \text { : } \\
\text { Explicación y análisis de estructura } \\
\text { prototípica } \\
\text { - Actividad 3: } \\
\text { Análisis de inicios de reseñas }{ }^{4} \\
\text { - Actividad 4: } \\
\text { Análisis de usos de adjetivos califi- } \\
\text { cativos } \\
\text { - Actividad 5: } \\
\text { Análisis de enunciados para formu- } \\
\text { lar temas } \\
\text { - Actividad } 6 \text { : } \\
\text { Análisis de forma y usos de marcas } \\
\text { lingüísticas de emisor y receptor } \\
\text { - Anexo. Actividades } 1-4 \text { : } \\
\text { Análisis de forma y usos de pregun- } \\
\text { tas retóricas }\end{array}$ & $\begin{array}{l}\text { - Planificación del } \\
\text { texto } \\
\text { - Textualización } \\
\text { - Revisión }\end{array}$ \\
\hline
\end{tabular}

Figura 1. Fases de la SD.

\section{Resultados}

Los resultados obtenidos a partir del análisis de datos giran en torno a tres aspectos: la relación entre los usos de las ayudas que ofrece la SD y las operaciones de escritura, los vínculos entre los usos de ayudas y las transformaciones que introducen los alumnos en sus textos y el proceso de apropiación del género como referencia durante la escritura.

\subsection{Las operaciones de escritura y el uso de las ayudas de la SD}

Los resultados del análisis de los datos sobre las operaciones de escritura (véase figura 2) y los usos de las ayudas que ofrece la SD muestran que:

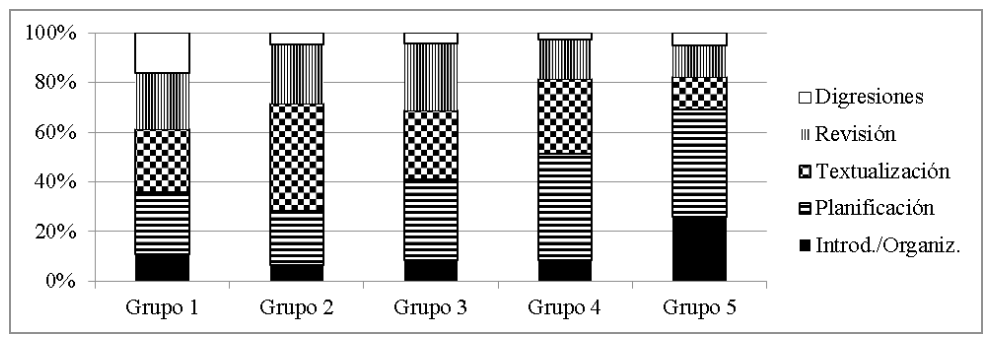

Figura 2. Las operaciones de escritura.

$4 \quad$ Rodríguez Gonzalo (2008) destaca la necesidad de plantear actividades que aborden los inicios de textos porque es el lugar en el que se gestiona la comunicación, porque facilita la lectura y porque ayuda a la planificación. La autora propone actividades de observación y análisis de diferentes posibilidades textuales, es decir, de valoración y comparación de modelos. 
1. Todos los grupos realizan todas las operaciones de escritura.

2. Los Gr. 1 y Gr. 3 dedican un porcentaje similar de intervenciones a cada operación. Estos son los grupos en los que se observa recursividad entre la planificación y la revisión durante la escritura y los que usan los modelos en fases más tempranas del proceso de elaboración textual (véase, más adelante, figura 5).

3. En el resto de grupos predomina una operación sobre las otras: en el Gr. 2 la mayoría de las intervenciones tienen como objetivo textualizar (43\%), mientras que en los Gr. 4 y Gr. 5 la mayoría de intervenciones está enfocada a planificar (43\%). En estos tres grupos el proceso de escritura es lineal (no hay recursividad de las fases) y no habrá una representación mental del texto a partir de los modelos de reseña.

4. Los Gr. 4 y Gr. 5 son los que tienen más dificultades durante la escritura y los que menos intervenciones dedican a la revisión (Gr. 4: 16\%; Gr. 5: 13\%) y más a la planificación $(43 \%)$.

\subsection{Las transformaciones textuales y los usos de ayudas durante el proceso}

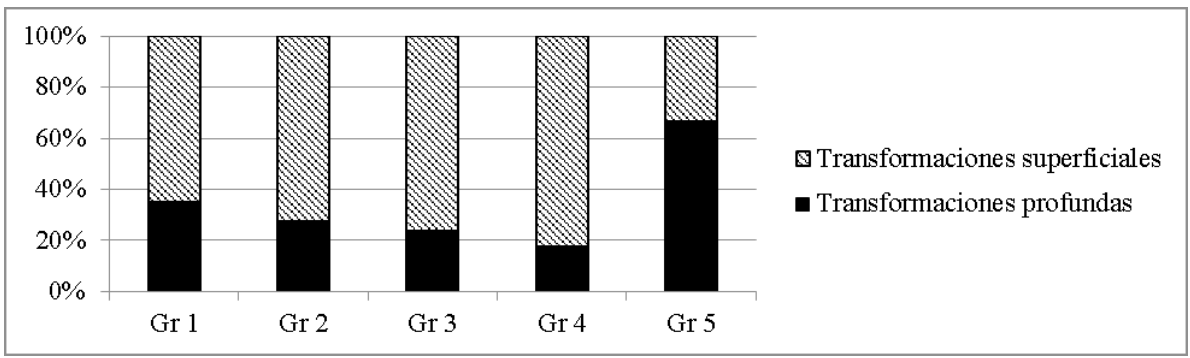

Figura 3. Las transformaciones textuales.

El análisis comparativo de los niveles textuales (véase figura 3) que están afectados por las transformaciones que realiza cada grupo permite observar que:

1. Los grupos que menos transformaciones de niveles profundos proponen durante la tarea de escritura son los Gr. 2 (27\%), Gr. 3 (23,53\%) y Gr. 4 (18\%). El Gr. 2 no se plantea conflictos durante la escritura de su texto que le lleven a reformular los planes iniciales. Sin embargo, elabora un texto correcto, aunque no ajustado a una reseña de lectura. El Gr. 3 reformula los planes iniciales, por lo tanto, introduce revisiones profundas en su escrito, pero el porcentaje de transformaciones de aspectos superficiales del texto $(76,47 \%)$ que propone durante el proceso es más alto que las profundas. El Gr. 4 tiene dificultades durante el proceso de escritura pero solo es consciente en el inicio de la textualización, en el único momento del proceso en el que utilizan los modelos de reseña como ayuda para escribir su texto (véase figura 4).

2. Las transformaciones textuales profundas (35\%) que propone el Gr. 1 durante el proceso de escritura son menores que las superficiales (65\%). Sin embargo, aunque introduzca menos transformaciones profundas, elabora un texto complejo y ajustado al género discursivo de la reseña de lectura (véase su texto final en la figura 6). 
3. El Gr. 5 es el grupo que más transformaciones textuales de niveles profundos $(66 \%)$ se plantea durante el proceso de escritura: estos alumnos son conscientes de sus dificultades durante la tarea de escritura, por lo que intentan resolver sus problemas en diferentes momentos del proceso.

4. El análisis de los momentos del proceso en los que se introducen las transformaciones (véanse las figuras 4 y 5) muestra que los modelos de reseñas activan transformaciones de aspectos profundos del texto en cuatro grupos: Gr. 1 (7 transformaciones), Gr. 3 (4), Gr. 4 (2), Gr. 5 (2) ${ }^{5}$. Por el contrario, hay pocos ejemplos en los que se observe que la plantilla de planificación active transformaciones profundas del texto

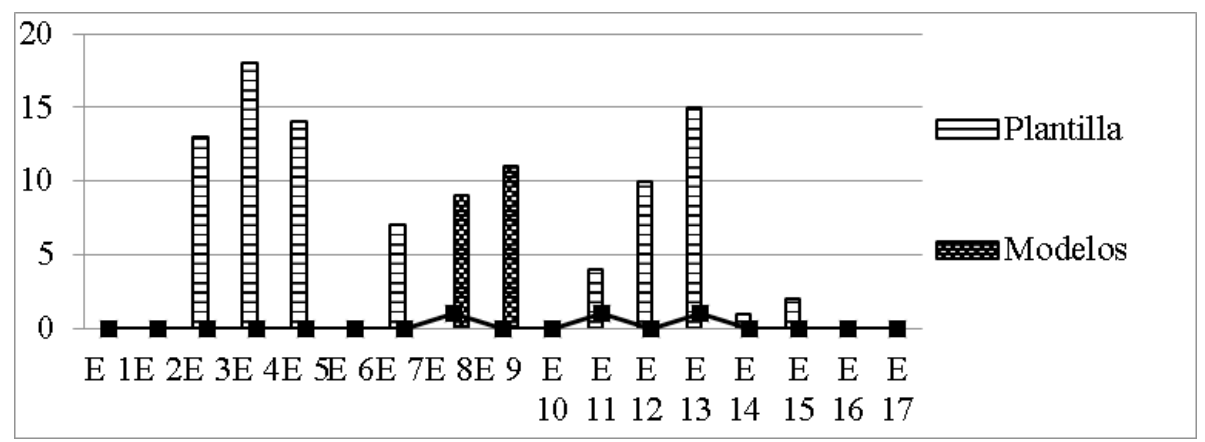

Figura 4. Transformaciones profundas del Gr. 4 y uso de modelos.

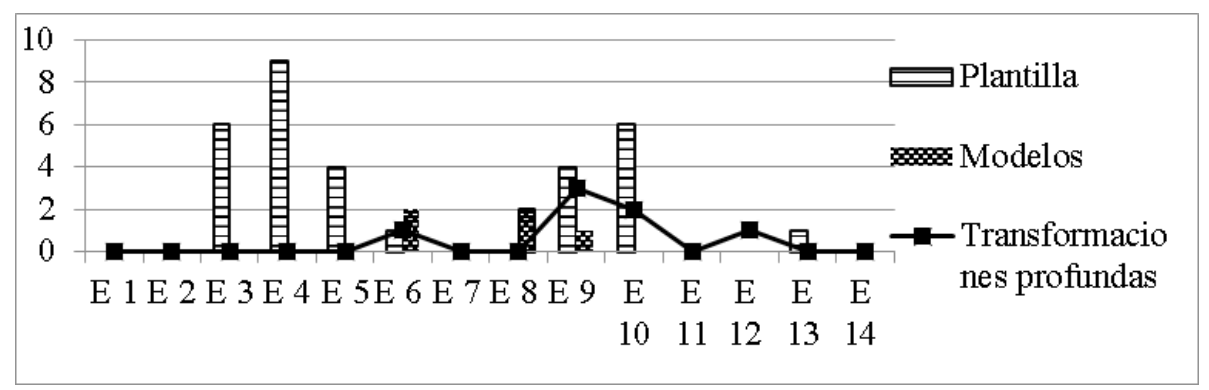

Figura 5. Transformaciones profundas del Gr. 1 y uso de modelos.

\subsection{El proceso de apropiación del género}

El análisis de los textos finales elaborados por los alumnos comparándolos con la plantilla de planificación que ofrece la SD y los modelos de reseña muestra que:

1. El Gr. 1 crea representaciones del género que le guían durante el proceso y concluye el proceso de apropiación del estereotipo (véase su texto final en la figura 6).

Solo un grupo, Gr. 2, no introducirá cambios de este tipo en su texto tras usar los modelos. En este caso estas transformaciones están formuladas por la alumna que tiene como referencia para la escritura un modelo de reseña. 
1960, Renewed 1988 py Harper Lee. Ediciones B, S. A., 2009 para el sello Zeta bolsillo. Barcelona (España)

Matar a un ruiseñor es una novela narrada por Scout, una niña valiente y algo curiosa que nos cuenta un tramo de su vida en el que su padre, Atticus, se ve obligado a enfrentarse a todo el pueblo por cumplir con la ley defendiendo a un negro.

¿Por qué no conseguimos dejar de leer esta novela? Porque, gracias a su lenguaje figurado en primera persona, Harper Lee ha conseguido cautivarnos, emocionarnos, e introducirnos en su fantástico libro. Por lo tanto creemos que tiene bien merecido el premio PULITZER que obtuvo en 1961.

Aunque su lectura nos ha resultado algo costosa porque poseía demasiados personajes conforme hemos ido leyendo nos hemos dado cuenta de que sería un tremendo error el no recomendar este libro, ya que, por su variada adaptación a todos los públicos cautivara a sus lectores.

Figura 6. Texto final Gr. 1.

2. El Gr. 3 no consigue apropiarse del estereotipo, aunque sí elabora una representación mental de la reseña que le guía durante la escritura.

3. Los Gr. 2, Gr. 4 y Gr. 5 no elaboran una representación mental del género porque no aíslan las características prototípicas de la reseña, por lo que no consiguen diferenciar este género de otros géneros. Esto les impide comparar el texto que escriben con el texto deseado y diagnosticar los problemas de su escrito. Aunque estos tres grupos no elaboran esta representación, se observan diferencias en el proceso de aprendizaje de los alumnos.

El Gr. 4 es el que tiene más dificultades. El hecho de que elabore un texto que reproduce las preguntas que aparecen en la plantilla (véase figura 7), y no un escrito descontextualizado y autónomo, obstaculiza aún más el establecimiento de características prototípicas que le permitan crear una representación mental de una reseña. Por ello, no pueden comparar su texto con esa representación mental y ser, de este modo, conscientes de los problemas que presenta su texto final.

\begin{tabular}{|c|c|c|}
\hline TEXTO FINAL & \multicolumn{2}{|c|}{ PLANTILLA DE PLANIFICACIÓN } \\
\hline $\begin{array}{l}\text { EL PUENTE DE LOS CEREZOS } \\
\text { Blanca Álvarez comenzó su trayectoria profesional } \\
\text { escribiendo en el diario } A S \text {, pasó a ser una gran es- } \\
\text { critora de novelas para jóvenes. Poniéndolo alto la } \\
\text { novela "El puente de los cerezos". } \\
\text { La obra valora la necesidad de mantener la comuni- } \\
\text { cación con la familia. } \\
\text { La protagonista vive muchos cambios en su vida pa- } \\
\text { sando de un mundo lleno de tecnología a otro ocupa- } \\
\text { do por la vida y las historias de sus antepasados [...]. } \\
\text { ¿Dónde ocurre la historia? } \\
\text { La historia ocurre en una aldea de campesinos de } \\
\text { China [...]. }\end{array}$ & $\begin{array}{l}\text { Obra y argumento: re- } \\
\text { flexiona sobre qué as- } \\
\text { pectos de la historia vas } \\
\text { a contar }\end{array}$ & $\begin{array}{l}\text { - Obra: año de publica- } \\
\text { ción, género, temas... } \\
\text { - } \quad \text { Quién (personajes): } \\
\text { - } \text { Qué (qué conflicto des- } \\
\text { encadena la historia de } \\
\text { la novela): } \\
\text { - Dónde y cuándo: }\end{array}$ \\
\hline
\end{tabular}




\begin{tabular}{|c|c|c|}
\hline $\begin{array}{l}\text { ¿Qué te enseña? } \\
\text { Te enseña como un día puedes estar en lo alto de la } \\
\text { vida y al día siguiente estar en lo más bajo, como } \\
\text { podemos observar en la frase: } \\
\text { "La vida tiene el peso de un cuenco de arroz" [...]. } \\
\text { La novela nos ha costado entenderla por los nombres } \\
\text { de los protagonistas, el paso que hace del presente al } \\
\text { pasado y los refranes que nos hacia reflexionar sobre } \\
\text { lo ocurrido. } \\
\text { Es una novela emocionante, sacrificada y sincera. }\end{array}$ & $\begin{array}{l}\text { Valoración: ¿Qué te ha } \\
\text { gustado más o menos? } \\
\text { ¿Qué te ha llamado la } \\
\text { atención? ¿Qué te ha re- } \\
\text { sultado difícil? ¿Qué te } \\
\text { ha sorprendido? }\end{array}$ & $\begin{array}{l}\text { - Personajes: } \\
\text { - Argumento: } \\
\text { - Lugar: } \\
\text { - Estilo y recursos litera- } \\
\text { rios: } \\
\text { Otros: }\end{array}$ \\
\hline $\begin{array}{l}\text { Se lo recomendamos a jóvenes estudiantes de secun- } \\
\text { daria y a adultos, ¿por qué se lo recomendamos a } \\
\text { ellos? Porque pensamos que ellos tienen más capa- } \\
\text { cidad de comprender los sentimientos que transmite } \\
\text { Lin-Lin. }\end{array}$ & Recomendación & $\begin{array}{l}\text { - ¿A quién lo recomen- } \\
\text { darías? } \\
\text { - ¿Por qué? }\end{array}$ \\
\hline
\end{tabular}

Figura 7. Comparación texto del Gr. 4 y plantilla de planificación.

El Gr. 5 elabora un texto autónomo pero alejado de una reseña de lectura. Este grupo tiene dificultades para crear una representación mental del género, pero al final de las sesiones de escritura, en el episodio en el que se elabora la ficha de revisión, los alumnos se percatan de su texto no se ajusta al género e intentan solucionar el problema.

El Gr. 2 no elabora una representación mental de una reseña de lectura pero consiguen escribir un texto correcto y autónomo que recoge el contenido de la SD.

\section{Conclusiones}

Nuestras conclusiones se agrupan en torno a cuatro aspectos:

1. Los datos muestran vínculos entre el uso de las reseñas de lectura como modelo de género y la recursividad o linealidad de las fases de escritura. En todos los casos se observa que los alumnos usan o pretenden usar los modelos para hacerse una representación mental del texto que deben realizar y que, cuando se usan los modelos con esta función, las operaciones de planificación y de revisión se interrelacionan.

No obstante, se observan diferencias en los procesos de escritura. Los alumnos que seleccionan una reseña como referencia para escribir su texto consiguen construir una representación mental del género que se activa durante diferentes momentos del proceso, lo que les ayuda a revisar y a reformular las planificaciones previas a partir de una comparación entre el texto que están elaborando y el texto deseado.

Por el contrario, los alumnos que tienen dificultades para elaborar una representación mental del género no revisan los planes iniciales, porque no alteran la primera representación del texto que surge de la plantilla de planificación que ofrece la SD. 
2. Los alumnos con dificultades para crear una representación mental del género acceden a evaluaciones de niveles complejos en su texto cuando vinculan la lectura y el análisis de las reseñas modelo con el texto que están elaborando. El acceso a una reflexión más compleja sobre sus escritos se debilita cuando dejan de usar esos modelos. Por el contrario, los alumnos que usan una reseña como referencia a lo largo del proceso introducen revisiones de estos niveles en diferentes momentos.

3. Los alumnos recurren a los modelos en la parte central del proceso. Todos los grupos los necesitan en el momento que inician la textualización: les permiten vincular la planificación, el género y el inicio de esta fase. Esto confirma que es uno de los momentos más complejos y difíciles de la escritura.

4. La comparación de los modelos de reseña que seleccionan los alumnos y los textos finales que elaboran muestra que hay alumnos que tienen más problemas para escribir un texto ajustado a este género porque no consiguen discriminar las características prototípicas y las periféricas de estos escritos. Por ello, no alcanzan el prototipo de las reseñas de lectura, sino un efecto de prototipo. Además, no logran abstraer las características que delimitan los géneros. Esta investigación evidencia que estos alumnos adaptan los modelos de género según las características que ellos atribuyen al género (no prototípicas). Por ello, pierden la conexión entre los modelos y los textos que están elaborando, hecho que dificulta el proceso para apropiarse del estereotipo.

\section{Bibliografía}

Abad, Victoria (2015): Los modelos de género discursivo en la planificación y revisión textual. Análisis del uso de modelos en alumnos de $3^{\circ} \mathrm{ESO}$ [en línea], Valencia, Universitat de València. http://roderic.uv.es/handle/10550/51937 [consulta: 16 agosto 2016]

Abril Villalba, Manuel (2006): "La escritura en la enseñanza de lenguas: investigación e instrucción", Lenguaje y textos, 23-24, 65-79.

Allal, Linda (2000): "Regulación metacognitiva de la escritura en el aula", en Camps, A. y Milian, M. (comps.): El papel de la actividad metalingüística en el aprendizaje de la escritura, Buenos Aires, Homo Sapiens, 187-214.

Álvarez Angulo, Teodoro (2006): "Didáctica de la escritura en la formación del profesorado", Lenguaje y Textos, 23-24, 47-62.

Álvarez Angulo, Teodoro (2010): Competencias básicas en escritura, Barcelona, Graó.

Álvarez Angulo, T. e I. García Parejo (2011): "Presentación: desarrollo de competencias escritas en los diferentes niveles del sistema educativo", Lenguaje y textos, 33, 5-8.

Amo, J. M. y M. del M. Ruiz Domínguez (2006): "Los géneros discursivos en la educación lingüística: la narración y la descripción”, Lenguaje y Textos, 23-24, 9-29.

Camps, A., O. Guasch, M. Milian, M. y T. Ribas (2007): "El escrito en la oralidad", Archivos de Ciencias de la Educación, 1(1). http://www.archivosdeciencias.fahce.unlp.edu.ar. [Consulta: 23 febrero 2014]

Camps, A. y T. Ribas (2000): La evaluación del aprendizaje de la composición escrita en situación escolar, Madrid, MECD.

Castelló, Montserrat (2000): "Las concepciones de los estudiantes sobre la escritura", en Camps, A. y M. Miliam (comps.): El papel de la actividad metalingüística en el aprendizaje de la escritura, Buenos Aires, Homo Sapiens, 67-104. 
Cuenca, M. J. y J. Hilferty (1999): Introducción a la lingüistica cognitiva, Barcelona, Ariel. Flower, L. y J. R. Hayes (1980): “The dynamics of composing: Making plans and juggling constraints”, en Gregg, L. W. y E. R. Steinberg (eds.), Cognitive processes in writing, Hillsdale (NJ), Lawrence Erlbaum Associates, 31-50.

Ganier, Franck (2006): "La révision des textes procéduraux", Langages, 164, 71-84.

Kervyn, Bernadette (2009): "Écriture poétique et fictionnalisation en fin d'école primaire", en Dufays, A. y S. Plane (coords.), La écriture de fiction en classe de français, Namur, Airdf / Presses Universitaires de Namur, 149-166.

Rodríguez Gonzalo, Carmen (2008): “La organización del aprendizaje lingüístico y literario. La unidad didáctica y los proyectos de trabajo", en Rodríguez Gonzalo, C. (ed.), La lengua escrita y los proyectos de trabajo. Propuestas para el aula, Valencia, Perifèric, 19-41.

Rodríguez Gonzalo, Carmen (2009): "La importancia de la planificación de los géneros discursivos en los alumnos de primaria y de secundaria y el diseño de tareas de escritura", Textos, 52, 97-107.

Scardamalia, M., C. Bereiter y H. Goelman (1982): “The Role of Production Factors in Writing Ability", en Nystrand, M. (ed.): What Writers Know, Nueva York, Academic Press, 173-210.

Zayas, Felipe (1997): "Las prácticas discursivas como eje para secuenciar las habilidades lingüísticas. Una propuesta para la ESO", Textos de Didáctica de la Lengua y de la Literatura, 11, 23-36.

Zayas, Felipe (2012): "Los géneros discursivos y la enseñanza de la composición escrita", Revista Iberoamericana de Educación, 59, 63-85. 